\title{
Why are non-steroidal anti-inflammatory drugs so variable in their efficacy? A description of ion trapping
}

\author{
G A Ellis, D R Blake
}

\begin{abstract}
Hypothesis
Our hypothesis is that acidic non-steroidal anti-inflammatory drugs (NSAIDs) undergo ion trapping in acidotic tissue and that this affects not only their gastrotoxicity, which is well known, but also their selectivity for, and their efficacy in, inflammatory joint disease.
\end{abstract}

Tissue acidosis may not be a feature of all chronic inflammatory disease states, but it does occur in inflammatory joint disease and is highly variable depending on the extent of the mismatch between synovial perfusion and metabolic demand. This mismatch is influenced not only by disease activity, but also by joint movement, which causes transient hypoxic episodes. Interventions, such as rest and drug treatment, would serve to reduce ion trapping and, therefore, NSAID efficacy through their effects on disease activity. The influence of these factors on $\mathrm{pH}$ dependent ion trapping may contribute to the variable efficacy of NSAIDs between disease states, between patients within a disease population, and within a single patient over time.

Prostaglandins have been found in increased amounts in many chronic inflammatory states, including rheumatoid arthritis ${ }^{1}$ and osteoarthritis, ${ }^{2}$ where they contribute to the severity and persistence of inflammation. They are derived from arachidonic acid by the action of cyclo-oxygenase and it is principally through the inhibition of this enzyme that NSAIDs exert therapeutic effects. ${ }^{3}$ Despite their mechanism of action NSAIDs are not universally effective in all chronic inflammatory disease states and this may be due, in part, to the pharmacokinetics of the drugs at the cellular level.

To reach their active site within inflammatory cells NSAIDs must be distributed to the extravascular compartment. Passage across the vascular endothelium can be either via gaps between the endothelial cells or by simple diffusion through them. There are, however, restrictions and both routes can be altered by disease.

Normally the gaps between endothelial cells are small, allowing only limited egress of small solutes, but restricting loss of protein. The presence of inflammation weakens the endothelial barrier, and the wider gaps allow the extravasation of water and hydrated molecules and proteins, including albumin. ${ }^{4}$
Non-steroidal anti-inflammatory drugs vary in their aqueous solubility, but most are highly albumin bound and will be co-distributed with this protein. Diffusion of an NSAID into the cell follows Fick's law, as it is dependent on the concentration gradient across the plasma membrane and the lipid/water partition coefficient of the drug molecule.

For weak acids, such as most NSAIDs, the effective concentration gradient is that of the non-ionised form, as ionised molecules are not lipid soluble

$$
\underset{\text { Non-ionised }}{\mathrm{HD}}=\underset{\text { Ionised }}{\mathrm{D}^{-}}+\mathrm{H}^{+}
$$

The position of the equilibrium depends on the intrinsic ionisation constant of the molecule (pKa) and on the $\mathrm{pH}$ value of the aqueous phase. The influence of $\mathrm{pH}$ is most profound around the $\mathrm{pKa}$ value of the molecule (fig 1).

For acidic NSAIDs, decreasing the $\mathrm{pH}$ increases the proportion of the non-ionised form, raises the overall lipophilicity, and enhances transport across the lipid membrane.

The $\mathrm{pKa}$ values for several NSAIDs at $25^{\circ} \mathrm{C}$ are given in the table. Within tissue, the actual values will vary slightly with temperature and the ionisation potential of the environment.

Where $\mathrm{pH}$ gradients exist across lipid membranes ionisable molecules are subject to ion trapping. The non-ionised form will tend to equilibrate across the membrane and will move down its concentration gradient into the high $\mathrm{pH}$ compartment where it will be ionised.

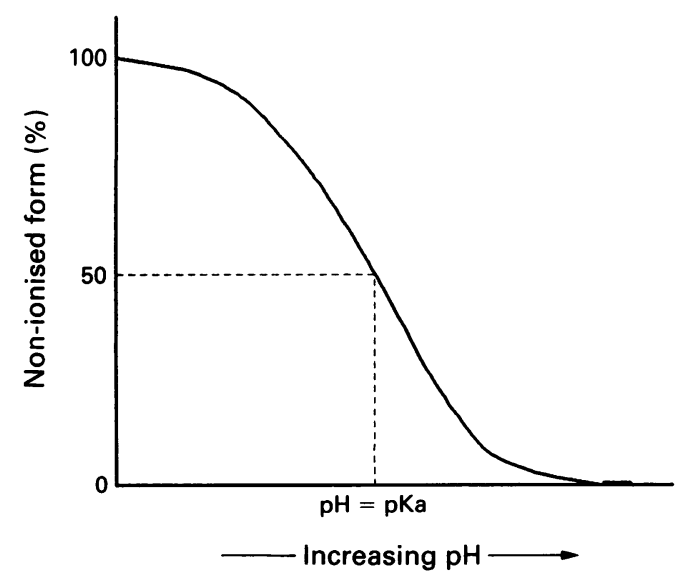

Figure 1 Ionisation of a weak acid. 
$p K a$ Values of various non-steroidal anti-inflammatory drugs (NSAIDs)

\begin{tabular}{ll}
\hline NSAID & $p$ Ka Value \\
\hline Salicylic acid & $3 \cdot 0$ \\
Diflunisal & $3 \cdot 3$ \\
Acetylsalicylic acid & $3 \cdot 5$ \\
Diclofenac & $3 \cdot 9$ \\
Mefenamic acid & $4 \cdot 2$ \\
Ibuprofen & $4 \cdot 4$ \\
Phenylbutazone & $4 \cdot 5$ \\
Indomethacin & $4 \cdot 5$ \\
Oxyphenbutazone & $4 \cdot 7$ \\
6-Methoxy-2-naphthylacetic acid & $5 \cdot 0$ \\
Piroxicam & $5 \cdot 1$ \\
\hline
\end{tabular}

^Active metabolite of nabumetone.

More non-ionised drug is then pulled across the membrane and this dynamic process continues until equilibrium is achieved (fig 2).

Ion trapping of NSAIDs within cells therefore occurs in situations where the $\mathrm{pH}$ of the extracellular fluid ( $\mathrm{pHe}$ ) is lower than that of the intracellular fluid (pHi), such as in the gastric mucosa and in acidotic diseased tissue. Normally the pHi of a cell is about $7 \cdot 1$ whereas the $\mathrm{pHe}$ is 7.5 . In vitro studies have shown, however, that the orientation of this $\mathrm{pH}$ gradient is reversed when the $\mathrm{pHe}$ decreases below approximately $7 \cdot 1$ in cells that have the mechanisms to cope with acidification..$^{56}$

In most situations the magnitude of the measurable pHe-pHi gradient will be small, but nevertheless it can have a considerable effect on the drug concentration at the active site. In human erythrocytes in vitro, decreasing the pHe of the phosphate buffer from $7 \cdot 4$ to $6 \cdot 8$ approximately doubled the concentration of phenylbutazone in the intracellular compartment, without affecting that of the basic compound, antipyrine. ${ }^{7}$ Physical effects can enhance the $\mathrm{pH}$ gradient. The electrostatic interaction between negatively charged groups in the plasma membrane reduces the $\mathrm{pHe}$ at the external surface of the cell. For example, with a bulk pHe of 7.0 the surface pHe is calculated to be only about $6 \cdot 2 .^{8}$ In addition, good buffering in the unstirred layer at the surface amplifies the ion trapping effect. Replacing sodium chloride in a buffer solution with sodium citrate produced a 43-fold increase in the ion trapping of salicylate. ${ }^{9}$

The gastric mucosa has the largest $\mathrm{pH}$ gradients in the body and is the prime site for NSAID trapping. These drugs accumulate in the cells of this tissue and produce a prolonged

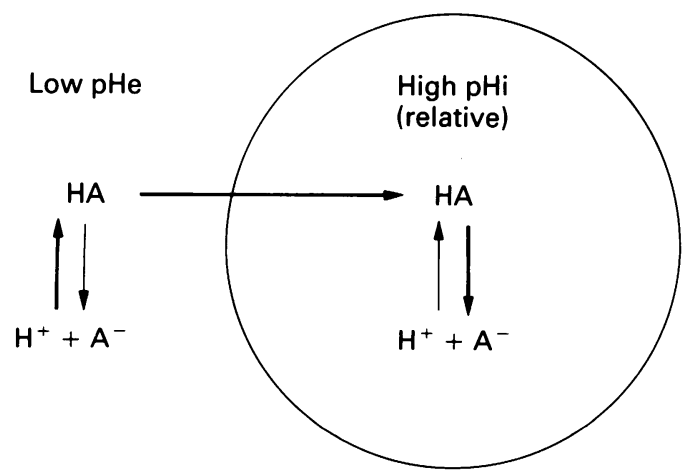

Figure 2 Ion trapping of weak acids. $p H e=p H$ of the extracellular fluid; $\mathrm{pHi}=\mathrm{pH}$ of the intracellular fluid. reduction in prostaglandins. ${ }^{10}$ Further down the intestinal tract $\mathrm{pH}$ gradients may occur but, if correctly orientated, are much smaller as the lumenal $\mathrm{pH}$ in these regions only varies between 7.0 and 8.0 . As a class the acidic NSAIDs have considerable gastrotoxicity and their reduction of endogenous prostaglandins is a major, but not the only, factor in this effect. ${ }^{11}$ It is interesting that the pattern of ion trapping parallels that of the gastrotoxicity profile. The incidence of endoscopically proved gastric ulcers in patients treated with NSAIDs is estimated to be about $20 \%,{ }^{12}$ whereas in the intestine the adverse effects of NSAIDs are generally limited to subclinical inflammation, and radiologically demonstrable ulceration and strictures occur only occasionally. ${ }^{13}$

McCormack and Brune ${ }^{14}$ considered the physicochemical characteristics of a few acidic NSAIDs with a lower than average gastrotoxic potential. They noted that these drugs did not reach high concentrations in the gastric mucosa. For flufenamic acid, fenclofenac, and diflunisal this was suggested to be owing to their enhanced lipophilicity, whereas for azapropazone it was thought to be because it is a cation at low $\mathrm{pH}$ values.

Ion trapping also occurs in tissues made acidotic by pathophysiological mechanisms such as in tumours and in inflammation. Research with ionisable 5-fluorouracil in rat fibrosarcomas in vivo, using nuclear magnetic resonance spectroscopy techniques, has shown that a localised pHe reduction from $7 \cdot 3$ to 6.9 enhanced the cellular uptake of the drug, causing a $2 \cdot 5$-fold increase in the elimination half life. ${ }^{15}$ Such direct studies have not been conducted in inflammation. Acidic NSAIDs have been shown to accumulate in inflamed tissue in several animal models, however. ${ }^{16}{ }^{17}$ In patients with joint inflammation, total NSAID concentrations increase in the synovial fluid and tissue as inflammation becomes more severe and, therefore, more acidotic. ${ }^{18} 19$ Although increased extravasation of albumin bound NSAIDs will contribute to the higher NSAID concentrations, ion trapping could also have a role.

If ion trapping does occur with NSAIDs, it would explain why acidic NSAIDs are more effective in vivo than NSAIDs such as proquazone and indoxole, which, although fairly potent cyclo-oxygenase inhibitors in vitro, are not acidic. ${ }^{20}$ It might also explain why more than $90 \%$ of patients with gout, with its severe synovitis, respond to NSAID treatment within one day, with complete resolution of symptoms in five to eight days ${ }^{21}$ and why the patients with rheumatoid arthritis most likely to respond well to NSAID treatment are those with more aggressive disease..$^{22}$ The acidosis of osteoarthritis is less severe and probably less persistent than in rheumatoid arthritis. ${ }^{23}$ Given this, NSAIDs would be expected to be most effective during inflammatory and acidotic episodes and less so in the intervening periods.

Differences in NSAID ion trapping may account for some of the variation in therapeutic response within a disease group, ${ }^{24}$ as acidosis 
is a dynamic variable and is under the influence of diverse factors. In rheumatoid arthritis the extent of acidosis differs considerably between patients and seems to be compounded by movement. ${ }^{192526}$ Flexion of an inflamed joint produces a small but significant decrease in $\mathrm{pH}$. This correlates with the flexion induced increase in intra-articular pressure, which, in turn, is accentuated by the presence of an effusion. ${ }^{27}$ Joint movement would, therefore, be expected to have a positive influence on the distribution of NSAIDs and may have contributed to the beneficial effects of physical training observed in patients with moderate rheumatoid arthritis. ${ }^{28} 29$ By the same token, any intervention, be it bed rest or drug treatment, that reduces disease activity would decrease NSAID ion trapping and therefore NSAID efficacy. In fact, NSAIDs could alter the synovial microenvironment in such a way as to attenuate their own efficacy over a period of time. The constantly changing profile of the influences on tissue $\mathrm{pH}$ could account for not only some of the variability between patients, but also that within a single patient over time.

The recently developed NSAID nabumetone uses the concept of ion trapping to good effect. Nabumetone itself is not acidic and is without significant pharmacological effects. It does not undergo ion trapping in the stomach and has low gastrotoxicity in animal models. ${ }^{10}$ It is metabolised in the liver to 6-methoxy-2-naphthylacetic acid, which is acidic, possesses similar anti-inflammatory activity to other acidic NSAIDs, and can potentially be ion trapped in inflammatory cells. ${ }^{30}$

In conclusion, we suggest that the variable efficacy of NSAIDs in different diseases, between patients for a selected disease, and within the same patient over time may be accounted for by the acidic nature of NSAIDs and their propensity for ion trapping. Detailed metabolic studies using nuclear magnetic resonance spectroscopy techniques that allow for accurate quantification of tissue $\mathrm{pH}$, and intracellular concentrations of suitably labelled NSAIDs, should be able to assess this hypothesis. In the meantime, should our hypothesis be true, clinical rheumatologists may like to consider some of the more obvious consequences. Movement of the joint may produce beneficial effects on drug distribution and, therefore, efficacy. Also, there may be a rationale for switching between NSAIDs, with their different physicochemical properties, to give continuing anti-inflammatory activity in a constantly changing microenvironment.

We thank the Arthritis and Rheumatism Council for their $\mathrm{P}$ Blower for his help with the manuscript.

1 Trang L E, Granstrom E, Lovgren O. Levels of prostaglandin $\mathrm{F} 2$-alpha and $\mathrm{E} 2$ and thromboxane $\mathrm{B} 2$ in joint fluid in rheumatoid arthritis. Scand $\mathcal{f}$ Rheumatol 1977; 6: 151-4.

2 Sahap-Atik O. Leukotriene B4 and prostaglandin E2-like activity in synovial fluid in osteoarthritis. Prostaglandins Leukot Essent Fatty Acids 1990; 39: 253-4.
3 Vane J R. Inhibition of prostaglandin synthesis as a mechanism of action for aspirin-like drugs. Nature 1971; 231: $232-5$.

4 Simkin P A, Pizzorno J E. Synovial permeability and rheumatoid arthritis. Arthritis Rhcum 1979; 22: 689-96.

5 Nuccitelli R, Heiple J M. Summary of the evidence and discussion concerning the involvement of $\mathrm{pHi}$ in the control of cellular functions. In: Nuccitelli R, Deame $\mathrm{D} \mathrm{W}$, eds. Intracellular $\mathrm{pH}$ : its measurement, regulation and utilization in cellular functions. New York: Liss, 1982 567-86.

6 Seisjo B K, Messeter K. Factors determining intracellular $\mathrm{pH}$. In: Seisjo B K, Sorensen S C, eds. Ion homeostasis of the brain. Copenhagen: Munksgaard, 1971:244-62.

7 Brune K, Graf P. Nonsteroidal anti-inflammatory drugs: influence of extracellular $\mathrm{pH}$ on biodistribution and pharmacological effects. Biochem Pharmacol 1978; 27 525-30.

8 Ohshima $\mathrm{H}$, Kondo T. $\mathrm{pH}$ dependence of electrostatic interaction between ion-penetrable membranes. Biophy Chem 1988; 32: 161-6.

9 Gutknetch T, Tosteson D C. Diffusion of weak acids across lipid bilayer membranes: effects of chemical reactions in the unstirred layers. Science 1973; 182: 1258-61.

10 Blower P. The scientific-equivalent efficacy and diminished risk. Eur F Rhumatol Inflamm 1991; 11: 29-37.

11 Whittle B J R. Temporal relationship between cyclooxygenase inhibition, as measured by prostacyclin biosvnthesis, and gastrointestinal damage induced by indomethacin in the rat. Gastroenterology 1981; 80: 94-8.

12 Gabriele S E, Bombardier C. NSAID induced ulcers. An emerging epidemic. f Rheumatol 1990;17: 1-4.

13 Bjarnason I, Zanelli G, Smith T, et al. Nonsteroidal antiinflammatory drug-induced intestinal inflammation in humans. Gastroenterology' 1987; 93: 480--9.

14 McCormack K, Brune K. Classical absorption theory an the development of gastric mucosal damage associated with non-steroidal anti-inflammatory drugs. Arch Toxicol 1987; 60: 261-7.

15 Guerquin-Kern J, Leteutre F, Croisy A, Lhoste J. pH dependence of 5 -fluorouracil uptake observed by in-vivo ${ }^{i 1} \mathrm{P}$ and ${ }^{19} \mathrm{~F}$ nuclear magnetic resonance spectroscopy. Cancer Res 1991; 51: 5770-3.

16 Brune K, Glatt M, Graf P. Mechanisms of action of antiinflammatory drugs. Gen Pharmacol 1976; 7: 27-33.

17 Brune K, Rainsford K D, Schweitzer A. Biodistribution of mild analgesics. Br f Clin Pharmacol 1980; 10: 279S-84S

18 Gaucher A, Netter P, Faure G, Schoeller J P, Gerardin A. Diffusion of oxyphenbutazone into synovial fluid synovial tissue, joint cartilage and cerebrospinal fluid. Eur 7 Clin Pharmacol 1983; 25: 107-12.

19 Falchuk K H, Goetzl E J, Kulka J P. Respiratory gases of synovial fluids. An approach to synovial tissue circulatory metabolic imbalance in rheumatoid arthritis. $\mathrm{Am} \mathcal{F} \mathrm{Med}$ 1970; 49: 223-31.

20 Brune $\mathrm{K}$, Otterness I. In-vivo and in-vitro assessment of non-steroidal anti-inflammatory drugs. Baillieres Clin Rheumatol 1988; 2: 295-307.

21 Fam A G. Strategies and controversies in the treatment of gout and hyperuricaemia. Baillieres Clin Rheumatol 1990, 4: $177-92$

22 Cush J J, Jasin H E, Johnson R, Lipsky P E. Relationship between clinical efficacy and laboratory correlates of inflammatory and immunologic activity in rheumatoid arthritis patients treated with non-steroidal antiinflammatory drugs. Arthritis Rheum 1990: 33: 623-33.

23 Naughton D P, Whelan M A, Grootveld M, Blake D R. New metabolic markers for arthritic disease etiology. In: Proceedings of the 55th American College of Rheumatology Meeting; 17-21 November 1991; Boston. S145.

24 Huskisson E C, Dieppe P A, Scott J, Jones H. Diclofenac sodium, diflunisal and naproxen: patient preferences fo anti-inflammatory drugs in rheumatoid arthritis. Rheumatol Rehabil 1982; $21: 238-42$.

25 Farr M, Garrey K, Bold A M, Kendall M J, Bacon P A Significance of hydrogen ion concentration in synovial fluid in rheumatoid arthritis. Clin Exp Rheumatol 1985; 3 : 99-104.

26 James M J, Cleland L G, Rofe A M, Leslie A L. Intraarticular pressure and the relationship between synovial perfusion and metabolic demand. $\mathcal{F}$ Rheumatol 1990; 17: perfusion

27 Merry P, Williams R, Cox N, King J B, Blake D R. A comparative study of intra-articular pressure dynamics in comparative study of intra-articular pressure dynamics in acute traumatic and chronic inflammatory joint effusions: potential implications for hyp
Rheum Dis 1991; 50: 917-20

28 Harkcom T M, Lampman R M, Banwell B F, Castor C W. Therapeutic value of graded aerobic exercise training in rheumatoid arthritis. Arthritis Rheum 1985; 28: 32-9.

29 Lyngberg K, Danneskiold-Samsoe B, Haskov O. The effec of physical training on patients with rheumatoid arthritis changes in disease activity, muscle strength and aerobic capacity. A clinically controlled minimized cross-ove study. Clin Exp Rheumatol 1988; 6: 253-60.

30 Haddock R E, Jeffery D J, Mangan F R, Pardon I S Penetration of nabumetone into the inflammator exudates in the rat. F Pharm Pharmacol 1983; 35: 358-62. 